\title{
Spectroscopy at Radio and Microwave Frequencies
}

\author{
D. J. E. INGRAM \\ M.A. (Oxon), D.Phil. (Oxon)
}

Lecturer and Research Fellow

University of Southampton

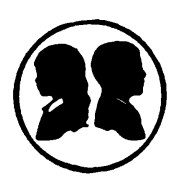

PHILOSOPHIGAL LIBRARY

NEW YORK 